\title{
Study on Protection Function of Quercetin on Ischemia-reperfusion Injury of Liver and its Mechanism
}

\author{
Qinhai Shen ${ }^{1, a}$, ZhaoMin Qin ${ }^{1,{ }^{*}}$, Ruixue Kong ${ }^{1}$ \\ ${ }^{1}$ Shandong Medical College, Jinan 250002 China \\ ashenqinhai@126.com \\ *Corresponding author qin888@163.com
}

Keywords: Quercetin; Ischemia reperfusion injury of the liver; Protect

\begin{abstract}
Objective: To report quercetin protection against hepatic ischemia-reperfusion injury and its mechanism were reviewed. Methods: The correlative literature retrieval in recent years, the quercetin on the protective effects of liver ischemia-reperfusion injury in experimental and clinical research in the case of analysis and summary. Results: The effect of quercetin is widespread, but by anti oxygen free radical and lipid peroxidation, inhibiting mitochondrial damage, intracellular calcium overload and cell apoptosis, improving microcirculation after reperfusion and anticoagulant mechanism of protecting ischemia-reperfusion injury of the liver. Conclusion: The quercetin has protective effect to liver ischemia-reperfusion injury, research and application value.
\end{abstract}

\section{Introduction}

Liver ischemia-reperfusion injury is a common pathological physiological changes during the process of liver surgery, can produce oxygen free radicals, the injury of liver cell ultrastructure, affect the function of the liver. The study found that the process of liver ischemia-reperfusion injury, the occurrence of apoptosis, is closely related to the role of oxygen free radicals. The liver ischemia-reperfusion injury is the partial liver transplantation, liver, blood loss and heart surgery and clinical diseases such as shock the common experience of a pathophysiological process. Research has shown that neutrophils after reperfusion are one of the main effectors cells of ischemia-reperfusion injury. The neutrophils and endothelial cell adhesion can enter into the liver parenchyma, many adhesion molecules involved in this process. ICAM - 1 (intercellular adhesion molecule - 1) molecules is one of the most important molecules. This study was to explore quercetin could inhibit liver blood sinus endothelial expression of intercellular adhesion molecule 1 and alleviate liver ischemia-reperfusion injury, from the level of molecular biology to reveal quercetin to sustain the mechanism of action of rat liver ischemia-reperfusion injury, for clinical further against ischemia-reperfusion injury to provide experimental basis.

\section{Materials and Methods}

\section{The animals and group}

The health, male Wistar rats, weight $220 \sim 220$ g, military academy of medical sciences animal center(Shandong university laboratory animal cente). Normal block material provided by the animal room to adapt to the feeding with weight according to random number table group after $1 \mathrm{w},(1)$ the normal control group; (2) liver ischemia reperfusion control group: the liver ischemia reperfusion group with the quercetin by gastric lavage average quantity solvent dimethyl sulfoxide (DMSO); (3) liver ischemia reperfusion group with the quercetin: tendency for $0.13 / \mathrm{kg}$ bw quercetin lavage solution, dissolved in DMSO, the doses used in the past research for us to improve the effective dose of peripheral blood total antioxidant force; (4) liver ischemia reperfusion group with the VC: tendency for $0.13 / \mathrm{kg}$ bw VC lavage, as the positive control group. 


\section{The experimental process}

The empty stomach is before the animal lavage for the night. $50 \mathrm{~min}$ after lavage, it is random death part of rats, liver determination of quercetin or VC content. In addition to the normal control group surgical ligation blood vessels not only, the rest are liver door after surgical ligation in rats blood vessels, loosen the blood vessels after $30 \mathrm{~m}$ in 30 min reperfusion, finally take the plasma was used to determine the relevant indexes and liver.

\section{The main reagent and instrument}

Bought quercetin from ACROS Company, double hydrogen chloride fluoresce in acetoacetate purchased from Sigma Company, the rest are homemade reagent; Beauty instrument one day type 8500 spectrophotometer, Hitachi F4500 fluorescence spectrophotometer, Waters 600 type highly effective liquid phase color spectrometer, etc.

\section{The pathological observation}

Light microscopy observation: shearing between groups of rats' myocardial ischemia area left ventricular anterior wall tissue, $10 \%$ formaldehyde fixed, conventional method preparation of paraffin section, HE stain after using organization structure change under observation. Electron microscope observation: rapid shearing between groups in the rat myocardial ischemia area left ventricular anterior wall tissue, fixed in $3 \%$ glutaraldehyde $2 \mathrm{~h}, 1 \%$ osmic acid fixed after $2 \mathrm{~h}$, dehydration after embedding the preparation of electron microscope thin slice, uranium acetate and lead citrate staining, double tissue ultrastructure change observed under transmission electron microscope.

\section{The statistical processing}

We use the SPSS19.0 with mathematical statistics software for data collection and statistical analysis, and chi-square test, $\mathrm{P}<0.05$, significant difference has statistical significance.

\section{Results}

\section{The influence of the quercetin on the content of quercetin liver}

The oxidative stress, high concentration of quercetin was shown to promote oxidation and the induced DNA damage. Observed through the experiment of $10 \mathrm{mu} \mathrm{mol} / \mathrm{L}$ under the concentration of quercetin can obviously reduce the $\mathrm{H}_{2} \mathrm{O}_{2}$ caused by peroxide product generation, but in the concentration of $200 \mu \mathrm{mol} / \mathrm{L}$, show some poisonous to the cells. Experimental results showed that ethanol infected liver cells in $50 \sim 75 \mu \mathrm{mol} / \mathrm{L}$ quercetin pretreatment appears the best effect of intervention, but when the dose of $100 \mu \mathrm{mol} / \mathrm{L}$ or more, the level of the CAT and alcohol group there was no significant difference. This shows that protection of quercetin with appropriate dose range, high dose may play a role of promote oxidation, there is a certain toxicity of liver cell.

\section{Discussion}

Quercetin can reduce the pathological damage of liver cells, such as the liver cell vacuoles degeneration, swollen mitochondria, rough endoplasmic reticulum expansion liver damage, reach the role of protecting liver, etc. Through the experimental observation of quercetin on carbon tetrachloride induced liver injury in rats. The results suggest that quercetin group of ALT, AST decreased significantly in the model group ( $\mathrm{P}<0.05$ ); Liver cell degeneration, necrosis and significantly reduce the inflammatory response. Experiment using the model of rat liver fibrosis induced by carbon tetrachloride, through the protein tyrosine kinase inhibitors, the interference of platelet derived growth factor (PDGF) receptor proliferation after the change of signal transduction pathways, observe the liver function and liver pathological changes. Experiment observed, liver damage period quercetin prevention of ALT, AST, a model group significantly reduce, liver cell damage degree is lighter than model group. At the same time, the inflammation pathological 
examination and grading showed quercetin prevention of liver cell degeneration, necrosis, and inflammatory cells infiltration degree lighter than the model group, shows that quercetin has certain protective effect on liver injury. In quercetin of trinitrotoluene (TNT) infected mice liver pathological morphology in the study of the influence of the experimental results show that TNT infected mice liver pathological changes in hepatic cell swelling, eosinophilic change and liver blood stasis for its outstanding characteristics. In TNT infected at the same time, to give oral quercetin in mice, significantly reduce visible liver damage degree, at the same time quercetin can also prompt damage to liver tissue of liver cell regeneration. The experimental results suggest that quercetin on TNT infected mice liver injury has certain protective effect.

\section{The neutrophils and liver ischemia-reperfusion injury}

The neutrophils gathered in the early stages of the liver ischemia-reperfusion injury to the liver microcirculation system is activated, increase the reperfusion injury. Neutrophils gathered outside invasion need hepatic sinus endothelial cells and the interaction between neutrophils, by neutrophils and endothelial cell surface adhesion molecules increase makes a combination of both more closely, and make further across the endothelial cells, neutrophils into the liver parenchyma, produce inflammation. The research thinks, the hepatic sinus neutrophils outside invasion is the main place, once outside invading the liver parenchyma, neutrophils related antigen by lymphocytes and liver cells intercellular adhesion molecules.

The change of mitochondrial structure and function has important relationship with the tissue damage, in the occurrence and development of IRI center link. The existence of the low concentration of reactive oxygen species is a normal physiological process, reactive oxygen species generated too much too fast will cause cell damage and lead to disease and aging. IRI after the production of large Numbers of oxygen free radicals, beyond the removal ability of antioxidant enzymes in mitochondria, the mitochondrial membrane lipid peroxidation increased, biological membrane structure is damaged, function is abate, viscera function is impaired.

Cause the release of the long time of protease and oxidative stress, the liver damage. Reperfusion, seeping into the organization of neutrophils in the role of NADPH oxidase produced a large number of oxygen free radicals; release the variety of proteolytic enzymes, damage to the liver cells and extracellular matrix. Kupffer cell calcium overload is its root cause is activated; the activation of Kupffer cells can participate in or mediated by release large amounts of toxic medium of liver damage. In addition the endothelial cells of calcium overload can result in intrahepatic microcirculation impedance increases, the lower the reperfusion microcirculation blood flow. With the deepening of the liver applied anatomy research and the development of various surgical instruments, hemostatic drugs improve the safety of the technique of liver resection and clinical practice Pringle has gradually reduce technique application. But the proper ischemic preconditioning may be effective protective measures to prevent the liver IR injury. Short of IR process cause organ damage more tolerance for subsequent more enduring IR technique known as ischemic preconditioning (is - chemicpreconditioning). Effect of ischemic preconditioning in brain, intestine, skeletal muscle and liver have confirmed that the A2 protection mechanisms may include adenosine, adenosine receptors, elimination of NO and TNF - a, the change of energy metabolism, maintenance of microcirculation and to accelerate the cell cycle and other factors.

The liver ischemia-reperfusion injury is often in the area of liver surgery involves a path physiological process. Neutrophils are the main effector's cells ischemia-reperfusion injury. Olanders use consumption such as neutrophils, neutrophils serum resistance and anti neutrophil adhesion of monoclonal antibody can reduce ischemia reperfusion leads to increased capillary permeability and capillary congestion, improve the function of viscera. Harada $\mathrm{N}$ application granulocyte activity such as inhibitors of rat liver was observed after ischemia-reperfusion, the drug can inhibit the neutrophil aggregation, reducing liver ischemia-reperfusion injury. Hepatic ischemia-reperfusion during early hepatic blood sinus endothelial cells and liver cells become cytotoxic effect of damage to a target, Kupffer cells become an important role in the early cytotoxic 
effect. In liver, ischemia, hypoxia first activate the intrahepatic mononuclear - system of Kupffer cells, macrophages secrete a variety of active substances and a large number of cytokines, such as tumor necrosis factor alpha (TNF alpha), IL - 1, etc.

\section{The quercetin on ischemia-reperfusion injury of liver protection}

Mitochondrial oxidative respiration is relied on the lining of the electron transport chain gradually implemented by electron transfer. Cytochrome $\mathrm{C}$ is a relative molecular weight on the mitochondrial respiratory chain of about 12,500 of water-soluble film peripheral protein, is easy to fall off from the film. Cytochrome $\mathrm{C}$ is on the eukaryotes mitochondrial respiratory chain is very important the electron transport body, its influence of variation of mitochondrial energy synthesis, thus the author chooses the cytochrome $\mathrm{C}$ as evaluation index of respiratory chain function. The model control group in ischemia-reperfusion after cytochrome $\mathrm{C}$ level significantly lower than the control group, mitochondrial ATP synthesis also appeared synchronization drop, suggests that mitochondrial electron transfer function of the destroyed, mitochondrial energy synthesis.

To study the mechanism of ischemia reperfusion and has become an important subject how to prevention and control of reperfusion injury. This experiment of animal model with left anterior descending coronary artery ligation of ordering the forming ischemic myocardial infarction, pine tied after coronary artery recanalization, close to human pathology after myocardial infarction, thrombolysis. The model was used to study the quercetin on the treatment mechanism of myocardial ischemia-reperfusion injury. Serum CPK is the specificity in the diagnosis of myocardial infarction (mi) isozyme, often as sensitive and specific indicators to judge and estimate the degree of myocardial infarction and prognosis. Quercetin can significantly reduce serum CPK, including quercetin high-dose group close to the control group. Quercetin low dose, middle dose and high dose group of CPK reduce degree is dose dependent $(\mathrm{P}<0.01)$. Showed that quercetin on ischemia-reperfusion rats myocardial damage has obvious protective effect. Lipid peroxidation mediated by free radical reaction is an important link in reperfusion injury. After reperfusion, the body produces free radicals, through various channels in the cell membrane lipid peroxidation and damage. GSH-Px is a kind of important free radicals in the body of the enzyme, in the balance of the body of the oxidation and anti-oxidation plays an important role. Literature reported that quercetin has to clear the role of oxygen free radicals. The experiment confirmed that quercetin in dose and high dose and verapamil can significantly improve the activity of GSH-Px. Hint of quercetin on ischemia-reperfusion myocardium damage protection and enhance the ability of myocardial antioxidant injury.

When the liver ischemia-reperfusion through the activation of $\mathrm{XO}$, mitochondria and microsome electronic messaging system is to generate a large number of oxygen free radicals, lipid peroxidation, and is causing the liver damage. We observe the liver ischemia reperfusion after serum GPT, GOT higher activity and MDA level, liver SOD, GSH-Px activity decreased, XO activity increases, elevated levels of MDA, GSH content decreased, suggesting that the liver ischemia reperfusion, enhanced oxidation reaction, and antioxidant defense function decline; We DCFH method was applied to observe the ROS produced the liver tissue ischemia reperfusion, the results showed ROS levels increased significantly. Although the rat livers can synthesis of VC, but liver ischemia-reperfusion after VC synthesis ability is impaired, causing the deterioration of VC content. It have already confirmed the $\mathrm{VC}$, such as Mr. Takayama on ischemia-reperfusion injury of liver protection, this study also got similar results. In serum GPT, GOT activity change, supplement the same dose of quercetin on ischemia-reperfusion injury of liver has a similar level of protection. In action segment, although both quercetin and $\mathrm{VC}$ after lavage can be generated in the process of clearing liver ischemia-reperfusion ROS, reduce consumption of important antioxidants GSH, raise TAOC, reduce the MDA generation, but quercetin protective ischemia-reperfusion injury of SOD, GSH-Px activity in the process of role, and VC had no obvious effect on two enzyme activity change, the specific reason remains to be further discussed.

Quercetin on the liver has the scavenging free radicals, ant oxidation, anti hepatitis b virus (HBV), liver cancer, liver fibrosis, protect liver damage, such as pharmacological action, is an effective 
active ingredient of many Chinese herbal medicine treatment of liver diseases. Now with quercetin as the main active ingredients of traditional Chinese medicinal materials such as cast yellow grass, yellow beads grass, water celery, etc., are widely developed for the treatment of jaundice hepatitis, hepatitis b liver disease such as drugs, and has set up through a variety of and clinical observation in vivo and in vitro, the results prove that has a definite therapeutic effect. Future of quercetin in the treatment of liver disease research, mainly through a variety of contain quercetin and the other has a therapeutic effect, the active ingredient of traditional Chinese medicine combined treatment of various liver diseases, and with the quercetin are gradually to clarify the mechanism of action of anti cancer of the liver, the liver tumor prevention and treatment will have broad prospect of application.

\section{Conclusion}

The DNA damage fracture phenomenon is a kind of cell apoptosis. The experimental results show that the $\mathrm{VC}$ on the liver ischemia-reperfusion in rats after liver cell DNA has significant control effect, however, quercetin has no, the specific reason is not clear. Above all, the quercetin lavage on ischemia-reperfusion injury after liver has obvious protective effect; the degree is similar to $\mathrm{VC}$, the mechanism of action is associated with the liver function of antioxidant system.

\section{References}

[1] Martinus I.F.J. Oerlemans, Stefan Koudstaal, Steven A. Chamuleau, Dominique P. de Kleijn, Pieter A. Doevendans, Joost P.G. Sluijter. Targeting cell death in the reperfused heart: Pharmacological approaches for cardioprotection[J]. International Journal of Cardiology. 2012

[2] Huanjun Pan, Dongye Li,Fang Fang,Dan Chen, Lingling Qi, Ruiqu Zhang,Tongda Xu,Hong Sun. Salvianolic Acid A Demonstrates Cardioprotective Effects in Rat Hearts and Cardiomyocytes After Ischemia/Reperfusion Injury[J]. Journal of Cardiovascular Pharmacology. $2011(5)$

trice Scazzocchio, Carmela Santangelo, Claudio Giovannini, Roberta Masella. Protocatechuic acid induces antioxidant/detoxifying enzyme expression through JNK-mediated Nrf2 activation in murine macrophages[J]. The Journal of Nutritional Biochemistry. 2011 (5)

[4] Hua-min ZHANG, Dan-li TANG, Lin TONG, Ming-jie SUN, Yu SUI, Hai-yan ZHU, Hong-xin CAO. Gualou Xiebai Banxia Decoction Inhibits NF-kappa B-dependent Inflammation in Myocardial Ischemia-reperfusion Injury in Rats[J]. Journal of Traditional Chinese Medicine. 2011 (4)

[5] Masanori Kawaguchi, Masafumi Takahashi, Takeki Hata, Yuichiro Kashima,Fumitake Usui, Hajime Morimoto,Atsushi Izawa, Yasuko Takahashi, Junya Masumoto, Jun Koyama, Minoru Hongo, Tetsuo Noda, Jun Nakayama, Junji Sagara, Shun'ichiro Taniguchi, Uichi Ikeda. Inflammasome Activation of Cardiac Fibroblasts Is Essential for Myocardial Ischemia/Reperfusion Injury[J]. Circulation . 2011 (6)

[6] Masafumi Takahashi. Role of the Inflammasome in Myocardial Infarction[J]. Trends in Cardiovascular Medicine. 2011 (2)

[7] Xin Wang, Xiao-long Ye,Rui Liu, Hong-Li Chen,Hua. Antioxidant activities of oleanolic acid in vitro: Possible role of Nrf2 and MAP kinases[J]. Chemico-Biological Interactions. 2010 (3)

[8] Aslan T. Turer, Joseph A. Hill. Pathogenesis of Myocardial Ischemia-Reperfusion Injury and Rationale for Therapy[J]. The American Journal of Cardiology. 2010 (3)

[9] Vilapakkam Ranganathan Punithavathi,Ponnian Stanely Mainzen Prince,Ramesh Kumar,Jemmi Selvakumari. Antihyperglycaemic, antilipid peroxidative and antioxidant effects of gallic acid on streptozotocin induced diabetic Wistar rats[J]. European Journal of Pharmacology. 2010 (1)

[10] K. Takada, T. Nakane,K. Masuda, H. Ishii. Ursolic acid and oleanolic acid, members of pentacyclic triterpenoid acids, suppress TNF- $\alpha$-induced E-selectin expression by cultured umbilical vein endothelial cells[J]. Phytomedicine. 2010 (14). 